ISSN 1112-9867

Available online at http://www.jfas.info

\title{
APPROXIMATION ALGORITHMS FOR GUARDING HOLEY POLYGONS
}

\author{
M. H. Moghaddam ${ }^{1}$, H. Divdel ${ }^{2}$, G. Alipour $^{3}$ \\ ${ }^{1}$ Department of Computer Engineering, Hashtrood Branch, Islamic Azad University, \\ Hashtrood, Iran \\ ${ }^{2}$ Department of Electrical Engineering, Tabriz Branch, Islamic Azad University, Tabriz, Iran \\ ${ }^{3}$ Department of Computer Engineering, Hashtrood Branch, Islamic Azad University, \\ Hashtrood, Iran
}

Published online: 15 May 2016

\begin{abstract}
Guarding edges of polygons is a version of art gallery problem. The goal is finding the minimum number of guards to cover the edges of a polygon. This problem is NP-hard, and to our knowledge there are approximation algorithms just for simple polygons. In this paper we present two approximation algorithms for guarding polygons with holes.
\end{abstract}

Keywords: guarding, approximation algorithm, vertex guard, edge guard.

Author Correspondence, e-mail: m.hosseinzadeh@iauhr.ac.ir

doi: http://dx.doi.org/10.4314/jfas.v8i3s.129

\section{INTRODUCTION}

In art gallery problem, one should find the minimum number of guards to patrol interior of a polygon. This means that, every point inside the polygon is visible from at least one of the guards.. The first result in this area, due to Chvatal, asserts that $\lfloor n / 3\rfloor$ guards are occasionally necessary and always sufficient to guard an art gallery represented by a simple polygon with $\mathrm{n}$ vertices [1]. In 1986 Lee and Lin proved that finding minimum vertex/point/edge guards are NP-hard [2]. These problems can not be approximated with an approximation ratio that is better than logarithmic in the number of polygon or terrain vertices for input polygons with 
holes or terrains. These problems can not be approximated with an arbitrarily small constant approximation ratio for input polygons without holes [3]. The best approximation algorithms for edge/vertex guards are presented by Ghosh [4].

see [1, 2, and 3] for comprehensive surveys. In some versions of the art gallery problem, one should find the minimum number of guards to patrol interior of a polygon. This means that every point inside the polygon is visible from at least one guard.. Guards may be placed inside or on the boundary of the polygon, and may be stationary or mobile. If stationary guards are restricted to be placed on the vertices of the polygon, they are called vertex guards; otherwise they are called point guards. Mobile guards that are restricted to move only along the edges of the polygon are called edge guards. A point is visible from an edge guard, if it is visible from at least one point of the edge on which the guard moves.

Laurentini [6] investigated a version of the art gallery problem, in which the guards should patrol the walls of the art gallery instead of patrolling its interior. This version was called edge-covering, and was compared to interior cover. He proved that the edge-covering problem is NP-hard. He introduced a restricted version of this problem, in which an edge should be completely visible from at least one point guard. He showed that this version is also NP-hard, and proposed an approximation algorithm with a logarithmic factor for a restricted point guard version of edge-covering problem.

Vertex and edge guard versions of edge-covering problem were solved by [5]. Their algorithms were designed only for simple polygons. In this paper we presented two approximation algorithms for guarding holey polygons.

\section{APPROXIMATION ALGORITHMS}

Suppose that $\mathrm{p}$ is a holey polygon with $\mathrm{m}$ holes and $\mathrm{n}$ vertices including holes vertices and $V P(P, z)$ denote the set of all points of $\mathrm{P}$ that are visible from a point $z \in P$. We call $V P(P, z)$ the visibility polygon of $\mathrm{P}$ from $\mathrm{z}$. A point $z \in P$ is said to be weakly visible from an edge $\mathrm{e}$ of $\mathrm{P}$ if there exists a point $\mathrm{u}$ on e such that the segment $\overline{z u}$ lies inside $\mathrm{P}$. Let $V P(P, e)$ denote the set of all points of $\mathrm{P}$ that are weakly visible from e. We call $V P(P, e)$ the weak visibility polygon of $\mathrm{P}$ from e. 


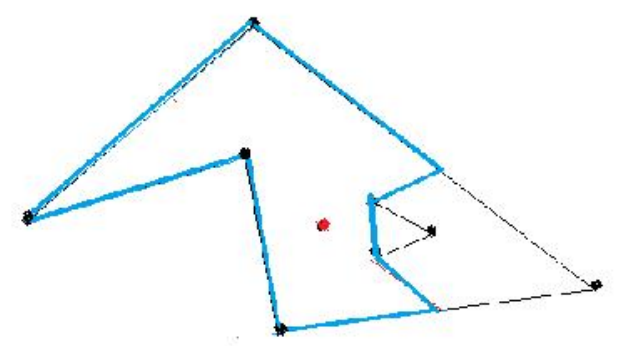

Fig.1. Visibility polygon of a point in a holey polygon with one hole

First, we consider the vertex guard version of the problem and present our approximation algorithm, and then we extend the proposed algorithm for the edge guard version. In the vertex guard version of the edge-covering problem, we should find the minimum number of vertex guards to patrol the edges of the given polygon. Passing lines through every pair of the vertices of the polygon we divide the edges of the polygon into some segments, are called the basic edge segments.

Definition 1. Edge segment $l_{1}$ on edge e is called basic edge segment, if there is no other edge segment $l_{2}$ such that $l_{1} \subset l_{2}$ and it can be divided by a line passing through the two vertices of the polygon.

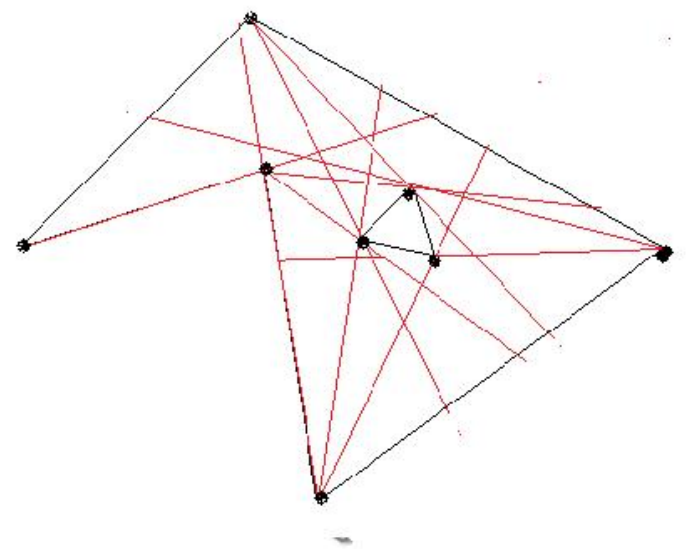

Fig.2. Basic Segments

Lemma 1. Every basic edge segment is completely visible from a vertex of the polygon.

Proof. Let us assume on the contrary that there is a basic edge segment $l$ that is partially visible from vertex $v_{j}$. Thus there is another vertex $v_{i}$ that line $\overline{v_{i} v_{j}}$ divides $l$, a contradiction. 
Thus, the vertex guard version of the edge-covering problem can be considered as an instance of the set covering problem [7]. The set that should be covered is the set of the basic edge segments, and the subsets are the sets of the basic edge segments that are visible from the vertices of the polygon. To find the basic edge segments, we compute the visibility polygons of the vertices, and find the intersections of their boundaries. Then we use Johnson's approximation algorithm for the set covering problem [8]. Since Johnson's algorithm has a logarithmic approximation factor, our algorithm also has. The algorithm is given by Algorithm 1. In the following we compute the time complexity of the algorithm. In step 1, computing the visibility polygon of each vertex of the given simple polygon takes $O(n)$ time [9]. Each visibility polygon consists of two types of edges, the edges that are on the boundary of the original polygon and the constructed edges that lie inside the original polygon. The basic edge segments are on the boundaries of the visibility polygons, and can be computed by finding the intersections of all the edges of the visibility polygons. Each visibility polygon has at most $O(n)$ edges on its boundary and there are $\mathrm{n}$ visibility polygons. Intersections of the edges of the two visibility polygons with $O\left(n_{1}\right)$ and $O\left(n_{2}\right)$ edges can be found in $O\left(n_{1}+n_{2}\right)$ time, and in the same time the edges of the two visibility polygons can be updated. This is due to this fact that the edges are segments of the edges of the original given simple polygon and are ordered according to its boundary. The union of two visibility polygons with $O\left(n_{1}\right)$ and $O\left(n_{2}\right)$ edges has $O\left(n_{1}+n_{2}\right)$ edges. Hence, the intersections of all the edges of all the visibility polygons can be computed in $O\left(n^{2}\right)$ time using a simple divide and conquer approach. step 2, we need to have all $F_{j}$ for all $1 \leq j \leq n$, which are the set of all the basic edge segments that are completely visible from $v_{j}$. These sets have already obtained in step 1 . Steps 3 to 7 are Johnson's approximation algorithm whose time complexity is $O(m n)$ [8]. Where $\mathrm{m}$ is the cardinality of the set that should be covered, i.e. the number of basic edge segments, and $\mathrm{n}$ is the number of subsets, i.e. the number of vertices of the given simple polygon. Thus the complexity of steps 3 to 7 is $O\left(n^{3}\right)$, this leads to a total time complexity of $O\left(n^{3}\right)$.

\section{Algorithm-1. Vertex guarding for the edge-covering}

1. Compute the visibility polygons of the vertices of the given simple polygon. Compute the basic edge segments by finding the intersections of the boundaries of the visibility 
polygons. Assuming $l_{1}, l_{2}, \ldots l_{m}$ are the basic edge segments, we define $E_{E}=\bigcup_{i=1}^{m} l_{i}$, which the set of the edges of the polygon is also.

2. For every $1 \leq j \leq n$, we define $F_{j}$ be the set of all the basic edge segments that are completely visible from $v_{j}$. Let $\mathrm{N}=\{1,2,3, \ldots, \mathrm{n}\}$, and $Q=\phi$

3. Find $i \in N$ such that $\left|F_{i}\right| \geq\left|F_{j}\right|$ for all $j \in N$ and $i \neq j$.

4. Add i to $\mathrm{Q}$ and delete $\mathrm{i}$ from $\mathrm{N}$.

5. For all $j \in N$, let $F_{j}=F_{j}-F_{i}, E=E-F_{i}$.

6. If $E \neq \phi$ go to 3 .

7. Output the set Q and stop.

Let consider the edge guard version of the edge-covering problem.

Lemma 2. Every basic edge segment is completely visible from an edge of the polygon.

Proof. Let us assume on the contrary that there is a basic edge segment $l$ that is partially visible from edge $e$. Thus there are two points a and $\mathrm{b}$ on $l$ such that edge $e$ sees a but not $\mathrm{b}$. Thus line segment $\overline{a b}$ intersects one of the constructed edges of the weak visibility polygon of edge $e$. Every constructed edge of a weak visibility polygon lies on a line passes through two vertices of the polygon. This means that a line passing through two vertices of the polygon divides a basic line segment, a contradiction.

Thus, the edge guard version of the edge-covering problem can be considered as an instance of the set covering problem. The set that should be covered is the set of the basic edge segments, and the subsets are the sets of the basic edge segments that are weakly visible from an edge of the polygon.

The proposed algorithm for vertex guard version can be simply modified for edge guard version. To this end, in the algorithm the weak visibility polygons of the edges should be used instead of the visibility polygons of the vertices. The weak visibility polygon of an edge of a simple polygon can be computed in linear time [10]. Thus the time complexity of the modified algorithm remains unchanged, and it is also $O\left(n^{3}\right)$.

\section{CONCLUSION}

In this paper we investigated the edge-covering problem for holey polygons. In this problem the guards should patrol the edges of a given holey polygon. We considered two versions of 
the problem, the vertex guard and the edge guard versions. We proposed two approximation algorithms with logarithmic factor for these two versions.

\section{REFERENCES}

[1] V. Chvatal, "A combinatorial theorem in plane geometry", Journal of Combinatorial Theory, vol. B 18, 1975, pp. 39-41.

[2] [2] D. T. Lee, , and A. K. Lin," Computational complexity of art gallery problems", IEEE Transactions on Information Theory, 1986, pp. 276-282.

[3] S. Eidenbenz, C. Stamm, and P. Widmayer," Inapproximability results for guarding polygons and terrains", Algorithmica, vol. 31, 2001, pp. 79-113.

[4] S. Ghosh, "Approximation algorithms for art gallery problems", Proc. of the Canadian Information Processing Society Congress, 1987, pp. 429-434.

[5] M.H. Moghaddam, A. Bagheri. CSICC2008,CCIS 6.pp.930-933,2008.

[6] A. Laurentini. Guarding the Walls of an Art Gallery, The Visual Computer 15, 265--278 (1999)

[7] T.H. Cormen, C.E. Leiserson, R.L. Rivest,. Introduction to Algorithms. 2nd edition, The MIT Press, Cambridge, Massachusetts, London, England, (2001)

[8] D.S. Johnson,. Approximation Algorithms for Combinatorial Problems. J. Computer and System Sciences 9, 256--278 (1974)

[9] H.A. EL Gindy, D. A. Avis, linear Algorithm for Computing the Visibility Polygon from a Point. J. Algorithms 2, 186--197 (1981)

[10] L. Guibas, J. Hershberger, D. Leven, M. Sharir, and R.E. Tarjan,. Linear Time Algorithms for Visibility and Shortest Path Problems inside Simple Polygons. In: 2nd ACM Symposium on Computational Geometry, pp. 1-13. ACM Press, New York (1986)

How to cite this article:

Moghaddam M H, Divdel H, Alipour G. approximation algorithms for guarding holey polygons. J. Fundam. Appl. Sci., 2016, 8(3S), 1063-1068. 